\title{
Systematic Review: Monoclonal Antibody-Induced Subacute Cutaneous Lupus Erythematosus
}

\author{
Chrissy Bolton 1,2,11 $\cdot$ Yifan Chen ${ }^{2} \cdot$ Rachel Hawthorne $^{3} \cdot$ lanthe R. M. Schepel $^{2}$ - Elinor Harriss ${ }^{4} \cdot$ Silke C. Hofmann $^{5}$. \\ Spencer Ellis ${ }^{6} \cdot$ Alexander Clarke $^{7} \cdot$ Helena Wace $^{8} \cdot$ Blanca Martin $^{9} \cdot$ Joel Smith $^{10}$
}

Published online: 22 September 2020

(c) The Author(s) 2020

\begin{abstract}
Background Subacute cutaneous lupus erythematosus (S(CLE) lacks consensus diagnostic criteria and the pathogenesis is poorly understood. There are increasing reports of SCLE induced by monoclonal antibodies (mAbs), but there are limited data on the aetiology, clinical characteristics and natural course of this disease.

Methods We devised a set of diagnostic criteria for SCLE in collaboration with a multinational, multispecialty panel. This systematic review employed a two-layered search strategy of five databases for cases of mAb-induced SCLE (PROSPERO registered protocol CRD42019116521). To explore the relationship between relative mAb use and the number of SCLE cases reported, the estimated number of mAb users was modelled from 2013 to 2018 global commercial data and estimated annual therapy costs.

Results From 40 papers, we identified 52 cases of mAb-induced SCLE, occurring in a cohort that was $73 \%$ female and with a median age of 61 years. Fifty percent of cases were induced by anti-tumour necrosis factor (TNF)-a agents. A median of three drug doses preceded SCLE onset and the lesions lasted a median of 7 weeks after drug cessation. Oral and topical corticosteroids were most frequently used. Of the licensed mAbs, adalimumab, denosumab, rituximab, etanercept and infliximab were calculated to have the highest relative number of yearly users based on global sales data. Comparing the number of mAb-induced SCLE cases with estimated yearly users, the checkpoint inhibitors pembrolizumab and nivolumab showed strikingly high rates of SCLE relative to their global use, but ipilimumab did not.

Conclusion We present the first systematic review characterising mAb-induced SCLE with respect to triggers, clinical signs, laboratory findings, prognosis and treatment approaches. We identify elevated rates associated with the use of checkpoint inhibitors and anti-TNFa agents.
\end{abstract}

\section{Introduction}

In an era of genomic medicine, the clear distinction of disease phenotypes is as critical as ever [1]. Integrated network analyses of-omics signatures are driving molecular disease classification that transcends organ-specific phenotypes [2]. However, a reductionist approach to disease classification retains its utility in the clinical setting and facilitates research [3]. Precise clinical phenotyping and confident diagnoses guide the initial therapeutic approach, provide an access key for interrogating large-scale datasets and make

Electronic supplementary material The online version of this article (https://doi.org/10.1007/s40268-020-00320-5) contains supplementary material, which is available to authorized users.

Chrissy Bolton

Christine.bolton@nhs.net

Extended author information available on the last page of the article

\section{Key Points}

Monoclonal antibody (mAb)-induced subacute cutaneous lupus erythematosus (SCLE) has been reported in 52 patients across a range of $17 \mathrm{mAbs}$.

Adalimumab, denosumab and rituximab were estimated to have the greatest number of annual users.

Checkpoint inhibitors and anti-tumour necrosis factor-a agents are associated with high rates of SCLE relative to the estimated global yearly use and other mAbs. 
data computationally accessible for machine learning analyses $[4,5]$. The phenotypic heterogeneity and overlap of autoimmune diseases provide a particular challenge with regard to clearly defining diagnostic criteria [6].

Lupus erythematosus (LE) exemplifies this classification challenge, in comprising a spectrum of autoimmune pathology, where aberrant inflammatory processes lead to cell injury with diverse patterns of disease. Cutaneous lesions may arise in isolation or as a feature of systemic lupus erythematosus (SLE), with a prevalence of around 70 in 100,000 [7]. In conventional classification, these cutaneous manifestations are classified as acute, subacute or chronic subtypes according to rash morphology, laboratory criteria and associated characteristics [8]. Such distinctions assist prognostication and predictions of whether patients are likely to manifest systemic features of LE [9].

Subacute cutaneous lupus erythematosus (SCLE) is a phenotypic subset of CLE, first described in 1979 as an intermediary between transient, acute CLE, and chronic, scarring CLE [10]. Forty years on, a consensus on the diagnostic criteria of SCLE remains elusive [11-13]. This lack of phenotypic clarity is exemplified in the pragmatic search methodology of previous reviews, where a published diagnosis of SCLE has been sufficient for inclusion rather than specified, standardised clinical criteria [14, 15]. With these considerations in mind, we aimed to clinically characterise and formalise the phenotype of SCLE to facilitate systematic review.

Numerous medications have been implicated in triggering the development of SCLE $[14,16]$, including monoclonal antibody $(\mathrm{mAb})$ products $[14,17]$ (hereafter referred to as 'mAb-induced SCLE' for simplicity). The clinical characterisation of mAb-induced SCLE has not been explored in systematic review of multiple databases. In particular, because of the specificity of mAbs and their immunomodulatory effects, patients with antibody-induced SCLE provide a unique perspective of the condition. Therefore, the aim of this review was to define and clinically characterise SCLE induced by $\mathrm{mAbs}$, to provide a clearer phenotype and prognosis. We then explored the relationship between relative $\mathrm{mAb}$ use on a global scale and the number of SCLE cases reported.

\section{Methods}

This review was conducted according to the PROSPERO registered protocol (CRD42019116521, accessible at https ://www.crd.york.ac.uk/prospero/display_record.php?Recor $\mathrm{dID}=116521)$ and the Preferred Reporting Items for Systematic Reviews and Meta-Analyses (PRISMA) guidelines.

\subsection{Eligibility Criteria}

A lack of formalised consensus for the classification criteria of SCLE [11-13] required the development of inclusion criteria by a multinational panel of dermatologists, rheumatologists and a dermatohistopathologist (Table 1). The agreed criteria incorporated morphological features specified by the European Society of Cutaneous Lupus Erythematosus (EUSCLE) [18] and previously published features of the disease $[14,19,20]$. To avoid ambiguity, the presence of lesions in sun-exposed regions was used to define 'photosensitivity'. A conservative boundary of 2 years post-initiation of therapy was used for inclusion as a drug-induced case [21]. Reports lacking sufficient detail to confirm inclusion criteria from three or more categories were excluded.

\subsection{Data Sources and Search Strategy}

Five databases were searched without any time limitations, with final searches conducted on 22 August 2019 (Fig. 1). The search strategy was designed in two layers using terms that included 'cutaneous', 'subacute', 'skin' and 'lupus' and terms for $\mathrm{mAb}$ therapies, including 'monoclonal antibody' and 256 named biologics (electronic supplementary Fig. S1). Alternative spellings, abbreviations and synonyms were included. Additional studies were identified from the review of abstracts from all 'Skin Immune-Mediated Inflammatory Disease' international conferences [22], manual searching of included study references, and correspondence with authors to identify missing data and obtain further patients from case series.

\subsection{Data Collection and Analysis}

Following duplicate removal, screening of abstracts, and critical appraisal, data extraction was performed by two reviewers independently using the same Microsoft Excel template (Microsoft Corporation, Redmond, WA, USA). Studies were eligible if they described human patients of any age and included individual-level primary data where sufficient patient characteristics could be extracted (electronic supplementary Table S1). English and non-Englishlanguage papers were included, with translation supplied by Google Translate for the initial screening. Variables extracted are specified in electronic supplementary Appendix S1. Verification of data extraction for German and Spanish papers was undertaken by bilingual doctors who were native speakers or had medical language qualifications in the language. Abstract screening disparities were resolved by a third reviewer, while appraisal and extraction disparities were resolved by discussion. 
Table 1 Inclusion and exclusion criteria used for monoclonal antibody-induced SCLE

\begin{tabular}{ll}
\hline Features & Inclusion \\
\hline Morphology & Definite: \\
& Annular, polycylic \\
& Erythema multiforme-like \\
& Papulosquamous, plaques or papules with scale or desquamation, psoriasiform \\
& 'SCLE' without exclusion terms \\
& Possible: \\
& Plaques or papules \\
& Erythema with scale or desquamation \\
& ANA-positive, anti-Ro antibody-positive OR ENA-positive \\
& Lesions in sun-exposed areas: \\
Face, neck OR arms & Prespecified: \\
-IF dermatitis \\
Photosensitivity \\
-Study synonyms: IF change, IF reaction, IF infiltrate, lichenoid reaction \\
-'Consistent with SCLE/lupus' \\
Supportive details included in cases: \\
-Lymphocytic infiltrate of perivascular/dermoepidermal regions/junction/peri- \\
follicular/superficial/deep/dermis/epidermis \\
-Parakeratosis, orthokeratosis, dyskeratosis, hyperkeratosis, necrotic/apoptotic \\
keratinocytes \\
-Mucin in dermis, colloid bodies, colloidal iron staining \\
Exclusion \\
Nonspecific/contradictory CLE terms in the absence of inclusion terms \\
Chronic, discoid, tumidus, panniculitis, profundus, chilblain, atrophic, scarring \\
Acute, malar, macular, butterfly \\
Stevens-Johnson syndrome \\
Palpable purpura, nodules, bullous \\
\hline
\end{tabular}

SCLE subacute cutaneous lupus erythematosus, ANA antinuclear antibody, ENA extractable nuclear antigen, $I F$ interface, $C L E$ cutaneous lupus erythematosus

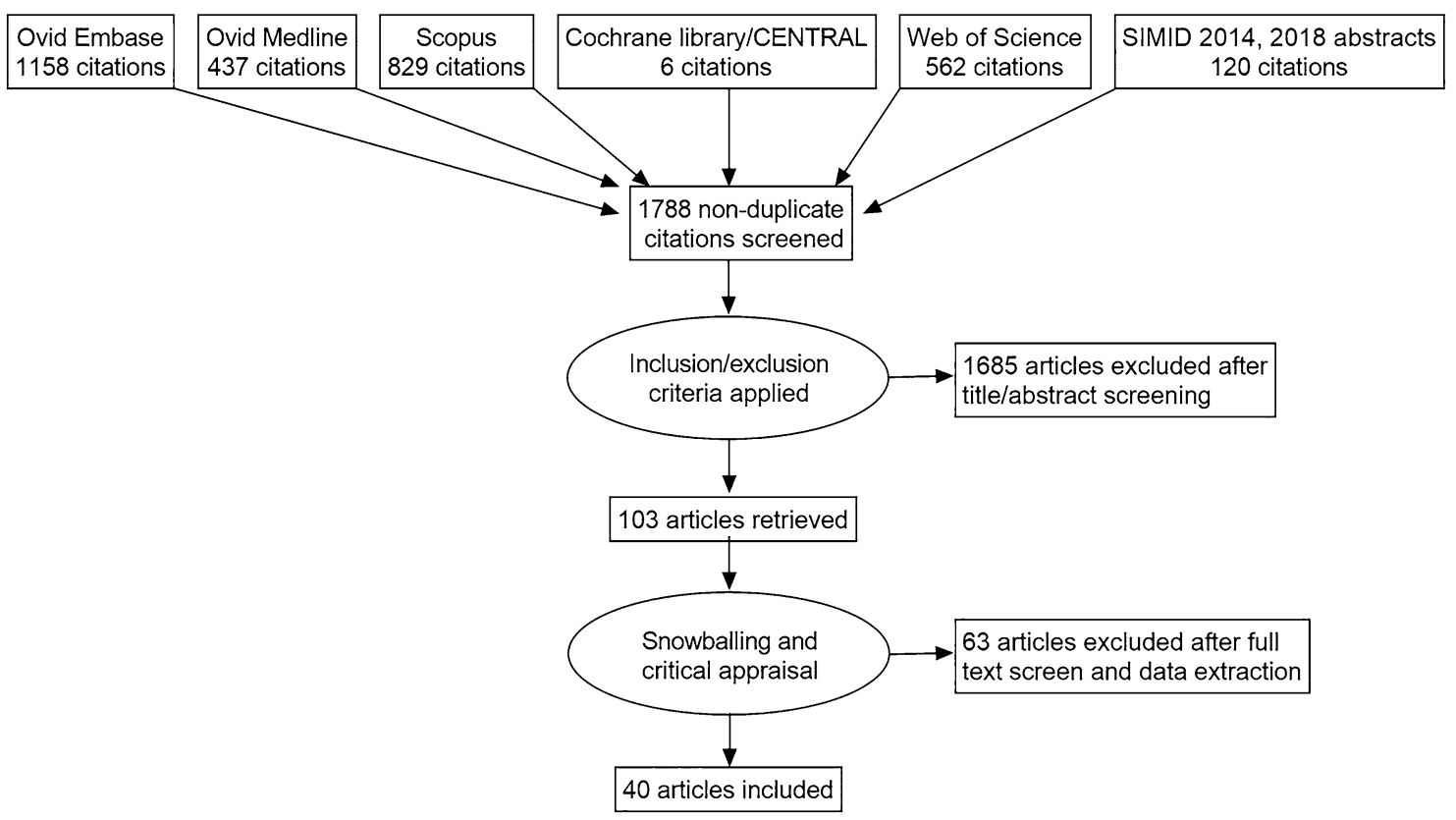

Fig. 1 Study profile of the systematic review for monoclonal antibody-induced subacute cutaneous lupus erythematosus 
Risk of bias was considered to be inherently low given the observational nature of case reports. The publication quality was appraised according to the accuracy of descriptions and figures, the consistency of values, and appropriate application of statistical tests (electronic supplementary Appendix S1).

\subsection{Monoclonal Antibody (mAb) Use}

To determine whether large-scale use of anti-tumour necrosis factor (TNF)-a agents accounted for high rates of SCLE, the relative global use of mAb products was estimated by modelling yearly global sales data (2013-2018) and dividing by the mean annual therapy cost taken from a truncated normal distribution of costs across different settings and indications. The $20 \mathrm{mAb}$ products with the highest global sales in 2013 (licenced in the US and Europe as of November 2013 [23]) were reviewed. These were updated to include the top $10 \mathrm{mAbs}$ with the highest sales in 2018 [24], amounting to 22 drugs. Biosimilars and profits from distributing companies for the US and the rest of the world were included in the total global sales from 2013 to 2018 (inclusive, values from 2018 shown in Table 3). Global sales were cross-checked with manufacturer financial reviews, additional studies and news reports [24, 25], and were assumed to follow a normal distribution, with fluctuations in global sales captured in the simulation model based on historical data from 2013.

A truncated normal distribution was used to estimate the average annual therapy costs with the upper bound based on US data, as the US is reported as having exceptionally high biologic costs [26]. 2017 and 2015 US published acquisition costs were extracted to derive the upper bound of annual treatment costs [27, 28] (electronic supplementary Table S2). For some mAb therapies, vastly disparate therapy costs were associated with differing indications [27]; therefore, a weighted average of costs was calculated according to the cost of each treatment regimen and the proportion of sales attributable to each indication (where provided by pharmaceutical companies [electronic supplementary Appendix S1 and Table S4]). The lower bound of annual treatment costs was based on the UK annual therapy costs using the UK National Institute for Health and Care Excellence (NICE) guidance on dosing and treatment costs [29] (electronic supplementary Table S3).

For the lower bound UK values, average costs were estimated for the treatment received by a single patient over 1 year, for adult indications, including induction and maintenance regimens. Yearly therapy prices were calculated for a range of oncological, musculoskeletal, respiratory and rheumatological indications of each drug as shown (electronic supplementary Table S3). Doses were calculated for a $70 \mathrm{~kg}$ adult with a body surface area of $1.75 \mathrm{~m}^{2}$. For drug treatments requiring a course duration of $<1$ year, the average duration of treatment was taken from NICE guidance where provided, or the median course of treatment from available clinical trials (electronic supplementary Table S3). UK treatment costs were derived from September 2015 vial prices (British National Formulary 70th edition), unless stated. UK costs in 2013-2018 Great British Pounds (GBP) were converted to 2018 US\$ using implied standard conversion rates based on purchasing power parities from the International Monetary Fund.

\subsection{Data Handling}

Descriptive statistics are provided according to information available in reports without adjustment. Regarding missing data, confidence intervals in Fig. 2 reflect proportions assuming missing data parameters were all positive or negative results. Statistical analysis using two-tailed Fisher's exact test was used where appropriate.

\section{Results}

\subsection{Case Inclusion}

Overall, 1788 unique abstracts from database searches and conference abstracts were screened for inclusion (Fig. 1). Reasons for exclusion were inability to extract patient data from aggregated cohorts, lesions occurring more than 2 years after $\mathrm{mAb}$ onset, an alternative diagnosis implied by rash description (Table 1), or reviews/expert opinion lacking clinical data. After manual searches, 103 full texts were critically appraised, with the inclusion of 40 studies. Reports of 52 individual patients had sufficient detail for inclusion (electronic supplementary Table S1), with inclusion criteria from at least three domains as described in Table 1; 69\% had a stated diagnosis of SCLE by the original paper authors.

\subsection{Patient Characteristics}

The median age of onset for mAb-induced SCLE was 61 years (interquartile range [IQR] 51-66) and $73 \%$ of patients were female $(38 / 52)$. The majority of patients had White European ancestry $(94 \%, 27 / 33)$. Inflammatory arthritis was the most common indication for mAb therapy at the time of the SCLE eruption $(40 \%, 20 / 50)$. The next most common indications for therapy leading to SCLE included advanced melanoma (12\%, 6/50), psoriasis/psoriatic arthritis $(10 \%, 5 / 50)$ and metastatic lung cancer $(8 \%, 4 / 50)$.

\subsection{Drug Characteristics}

mAbs implicated in inducing SCLE are shown in Table 2. The median time to onset after initiating mAb therapy was 9 weeks (IQR 3-17) [Table 2]. Given the weekly or monthly 

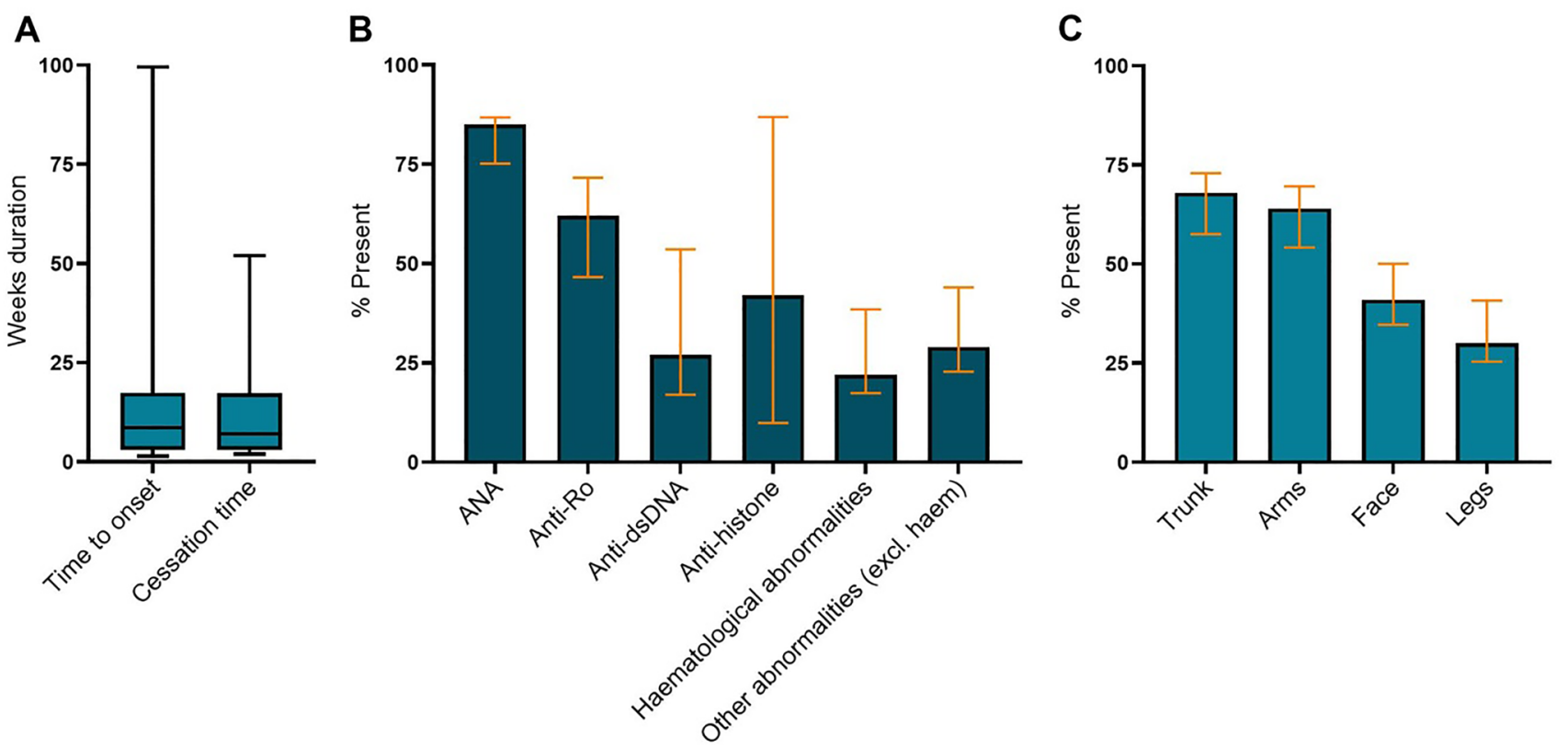

Fig. 2 a Time course and $\mathbf{b}$, $\mathbf{c}$ proportion of monoclonal antibodyinduced subacute cutaneous lupus cases demonstrating particular clinical features. Orange confidence intervals illustrated in $\mathbf{b}, \mathbf{c}$ show the potential range of results if all missing data were a positive or negative result. ANA antinuclear antibody

sun avoidance and ultraviolet (UV) protection were generally unambiguously applied; therefore, we focused on therapeutics pertinent to clinical decision making. Sixteen percent of patients $(8 / 49)$ were treated with biologic cessation alone as first-line. One of these patients started the DMARD hydroxychloroquine, however the rash had already started to improve [30]. Some patients did not initially stop their $\mathrm{mAb}$ therapy until the drug-induced nature of the rash was suggested; two of three patients did not see improvement until cessation of the drug despite starting topical corticosteroid creams at rash onset [31, 32]. For 38 of the remaining patients, mAbs were stopped in combination with other therapies.

The most common medications applied were oral and/or topical corticosteroids. Thirty-one percent of patients were treated with topical corticosteroids alone (15/49), with triamcinolone $0.1 \%$ ointment being the most frequently used topical corticosteroid. Thirty-seven percent of patients were treated with oral corticosteroids (18/49), either alone $(n=5)$ or in combination with disease-modifying antirheumatic drugs (DMARDs; $n=4)$, topical corticosteroids $(n=3)$, both these therapies $(n=5)$ or other immune-modulating therapies $(n=1)$. One patient received infliximab and oral prednisolone, but infliximab was discontinued after 5 months due to a lack of response [33]. With sun protection and topical corticosteroids added, the skin eruptions of this patient resolved within 1 month [33]. Thirty percent of patients received DMARDs (15/49) alone or in combination. The DMARDS used first-line included hydrochloroquine were recorded $(n=49)$ [Table 2]. Preventative measures of 
Table 2 Summary of patient characteristics experiencing monoclonal antibody-induced subacute cutaneous lupus erythematosus

\begin{tabular}{|c|c|c|c|c|}
\hline Trait & Percentage & Further detail & Monoclonal antibody & No. of SCLE cases \\
\hline Age, years $(n=52)$ & $\begin{array}{l}\text { Median } 61 \\
\text { Mean } 58.9\end{array}$ & $\begin{array}{l}\text { IQR 51-66 } \\
\text { Range 28-82 }\end{array}$ & $\begin{array}{l}\text { Etanercept } \\
\text { Adalimumab }\end{array}$ & $\begin{array}{l}10 \\
6\end{array}$ \\
\hline Sex & $73 \%$ female & $38 / 52$ & Infliximab & 6 \\
\hline Ethnicity & $\begin{array}{l}\text { 94\% White } \\
\text { 3\% Omani } \\
3 \% \text { Brazilian }\end{array}$ & $\begin{array}{l}27 / 33 \\
1 / 3 \\
1 / 33\end{array}$ & $\begin{array}{l}\text { Nivolumab } \\
\text { Pembrolizumab } \\
\text { Golimumab } \\
\text { Bevacizumab }\end{array}$ & $\begin{array}{l}6 \\
6 \\
4 \\
2\end{array}$ \\
\hline Onset time, weeks $(n=47)$ & $\begin{array}{l}\text { Median } 9 \\
\text { Mean } 13.8\end{array}$ & $\begin{array}{l}\text { IQR 3-17 } \\
\text { Range 1-100 }\end{array}$ & $\begin{array}{l}\text { Abatacept } \\
\text { Rituximab }\end{array}$ & $\begin{array}{l}2 \\
2 \\
2\end{array}$ \\
\hline Cessation time, weeks $(n=33)$ & $\begin{array}{l}\text { Median } 8 \\
\text { Mean } 14\end{array}$ & $\begin{array}{l}\text { IQR 3-17 } \\
\text { Range 2-52 }\end{array}$ & $\begin{array}{l}\text { Denosumab } \\
\text { Efalizumab }\end{array}$ & $\begin{array}{l}1 \\
1\end{array}$ \\
\hline Lesion distribution $(n=44)$ & $\begin{array}{l}68 \% \text { trunk } \\
64 \% \text { arms } \\
41 \% \text { face } \\
30 \% \text { neck } \\
30 \% \text { legs } \\
30 \% \text { back } \\
18 \% \text { hands } \\
14 \% \text { head }\end{array}$ & $\begin{array}{l}30 / 44 \text { trunk } \\
28 / 44 \text { arms } \\
18 / 44 \text { face } \\
13 / 44 \text { neck } \\
13 / 44 \text { legs } \\
13 / 44 \text { back } \\
8 / 44 \text { hands } \\
6 / 44 \text { head }\end{array}$ & $\begin{array}{l}\text { Natalizumab } \\
\text { Ranibizumab } \\
\text { Secukinumab } \\
\text { Ixekizumab } \\
\text { Atezolizumab } \\
\text { Ustekinumab } \\
\text { First-line treatment }(n=49)\end{array}$ & $\begin{array}{l}1 \\
1 \\
1 \\
1 \\
1 \\
1\end{array}$ \\
\hline Serology & $\begin{array}{l}85 \% \text { ANA }+ \\
62 \% \text { anti-Ro }+ \\
27 \% \text { anti-dsDNA } \\
42 \% \text { anti-histone }\end{array}$ & $\begin{array}{l}39 / 46 \\
24 / 39 \\
9 / 33 \\
5 / 12\end{array}$ & $\begin{array}{l}\text { Monotherapy: } \\
\text { Biologic cessation } \\
\text { TOP corticosteroid only } \\
\text { PO corticosteroid only } \\
\text { DMARD only }\end{array}$ & $\begin{array}{l}16 \%(8 / 49) \\
31 \%(15 / 49) \\
10 \%(5 / 49) \\
8 \%(4 / 49)\end{array}$ \\
\hline Haematological & $\begin{array}{l}\text { Pancytopenia } \\
\text { Lymphopenia } \\
\text { Leukopenia } \\
\text { Low complement }\end{array}$ & $\begin{array}{l}2 / 52 \\
3 / 52 \\
4 / 52 \\
4 / 52\end{array}$ & $\begin{array}{l}\text { Polytherapy: } \\
\text { TOP + PO corticosteroid } \\
\text { DMARD + PO corticosteroid } \\
\text { DMARD + TOP corticosteroid } \\
\text { DMARD + PO + TOP corticosteroid } \\
\text { Other }\end{array}$ & $\begin{array}{l}6 \%(3 / 49) \\
8 \%(4 / 49) \\
2 \%(1 / 49) \\
10 \%(5 / 49) \\
8 \%(4 / 49)\end{array}$ \\
\hline
\end{tabular}

$A N A$ antinuclear antibodies, $P O$ per oral, TOP topical, DMARD disease-modifying antirheumatic drug, IQR interquartile range, $S C L E$ subacute cutaneous lupus erythematosus, $d s D N A$ double-stranded DNA

( $n=10)$, chloroquine $(n=2)$, mycophenolate $(n=2)$ and quinacrine $(n=1)$, making hydroxychloroquine the most used DMARD. A higher proportion of patients started on DMARD therapy had more clinical findings reported (53\%, $n=8 / 15)$ than those not receiving DMARDs $(15 \%, n=4 / 26)$. Only one patient received a calcineurin inhibitor as part of therapy [34].

\subsection{Prognosis}

Eighty-nine percent of patients were reported to respond partially or fully to first-line therapy (30/45). One patient experienced a flare when corticosteroids were weaned, and again when hydroxychloroquine was introduced as a corticosteroid-sparing agent [35]. Ten patients went on to have second-line therapy or dose adjustments for their mAbinduced SCLE due to non-response or partial response. Most commonly, a DMARD was added for patients receiving corticosteroid therapies; $9 / 10$ of these patients responded to second-line therapy and recovered. Overall, where SCLE lesions responded to first-line therapy with complete or partial resolution, it took a median of 3.5 weeks (IQR 3-12, $n=26$ ). Alongside sun protection, patients who were managed by stopping $\mathrm{mAb}$ therapy alone saw improvement or resolution of skin lesions within a median of 3 weeks (IQR $3-3.8, n=6)$. Seventy-five percent of patients (9/12) receiving topical corticosteroids alone showed response to this therapy within 3 weeks. For all patients, it took a median of 8 weeks for lesions to resolve (IQR $3-17, n=33$ ).

\section{6 mAb Use}

In 2013, the mAbs with the highest sales were adalimumab, infliximab, etanercept, rituximab and bevacizumab. By 2018, the highest sales were attributed to adalimumab, etanercept, pembrolizumab, trastuzumab and bevacizumab. From modelling 2013-2018 global sales against estimated yearly therapy costs, the mAbs with the estimated highest yearly number of users were adalimumab, denosumab, rituximab, etanercept and infliximab (Table 3).

With a high average therapy cost, pembrolizumab had 68,000 estimated yearly users, compared with adalimumab 
with an estimated 615,000 yearly users. The number of SCLE cases attributable to checkpoint inhibitors such as pembrolizumab and nivolumab showed an especially high ratio of SCLE relative to the estimated yearly users (Fig. 3). Pembrolizumab and nivolumab target programmed celldeath-1 (PD-1) antigens, while another checkpoint inhibitor ipilimumab targets cytotoxic T-lymphocyte-associated antigen-4 (CTLA-4). In contrast, ipilumumab was not associated with any cases but showed reduced numbers of estimated yearly users compared with pembrolizumab and nivolumab. The anti-TNFa agents included (adalimumab, etanercept, infliximab, golimumab and certolizumab pegol) show an elevated rate of SCLE relative to the estimated yearly users, especially when compared with other highly used drugs such as denosumab and rituximab. The exception was certolizumab pegol, which is a modified fragment of an $\mathrm{mAb}$ and is estimated to have many fewer annual users than other antiTNFa agents (Table 3). Ustekinumab targeting interleukin (IL)-23 and IL-12, and omalizumab targeting immunoglobulin (Ig) E Fc regions were not associated with SCLE cases despite a high number of estimated users (approximately 190,000 and 121,000 , respectively).

\section{Discussion}

To the best of our knowledge, this systematic review is the first to explicitly define clinical criteria for SCLE to robustly determine the inclusion of patients. With increasing $\mathrm{mAb}$ use and rising rates of drug-induced SCLE (DI-SCLE) [14], we systematically reviewed for the first time the clinical course and treatment approach for mAb-induced SCLE. To derive the rates of SCLE relative to the estimated number of users of $\mathrm{mAb}$, we modelled estimated yearly users from global sales and average treatment costs across a number of indications.

\subsection{Inclusion Criteria}

The devised scoring criteria combined multiple clinical factors to reflect the diagnostic approach to SCLE $[18,19,36]$.

Table 3 Estimated monoclonal antibody use according to total global sales and average global cost

\begin{tabular}{|c|c|c|c|}
\hline $\mathrm{mAb}$ & $\begin{array}{l}\text { Total global sales in } 2018 \\
(\mathrm{US} \$, \$ \mathrm{~m})^{\mathrm{a}}\end{array}$ & $\begin{array}{l}\text { Mean global mAb cost }[\text { US\$ (min- } \\
\max )]^{b}\end{array}$ & $\begin{array}{l}\text { Average estimated mAb yearly users across } \\
\text { the years } 2013-2018 \text { [in thousands (min- } \\
\max \text { )] }\end{array}$ \\
\hline Adalimumab & 19,952 & $34,870(14,141-55,614)$ & $615(331-1524)$ \\
\hline Etanercept & 7611 & $30,105(11,085-49,142)$ & $276(149-713)$ \\
\hline Pembrolizumab & 7171 & $107,625(80,214-135,072)$ & $68(40-109)$ \\
\hline Trastuzumab & 7053 & $47,119(19,869-74,389)$ & $160(90-367)$ \\
\hline Bevacizumab & 6919 & $86,810(36,383-137,278)$ & $84(49-193)$ \\
\hline Rituximab & 6821 & $23,559(6323-38,153)$ & $305(172-1102)$ \\
\hline Aflibercept & 6746 & $38,425(9378-67,491)$ & $187(77-818)$ \\
\hline Nivolumab & 6735 & $102,425(40,614-164,291)$ & $70(37-179)$ \\
\hline Infliximab & 6593 & $22,790(15,730-29,854)$ & $297(209-440)$ \\
\hline Ustekinumab & 5156 & $28,586(15,280-42,415)$ & $190(86-435)$ \\
\hline Denosumab & 4077 & $13,069(3804-22,341)$ & $325(131-1286)$ \\
\hline Ranibizumab & 3722 & $32,341(10,187-54,514)$ & $125(63-396)$ \\
\hline Eculizumab & 3563 & $547,771(509,185-586,412)$ & $7(3-10)$ \\
\hline Golimumab & 2977 & $33,712(15,244-52,192)$ & $94(51-216)$ \\
\hline Omalizumab & 2970 & $26,148(12,696-39,619)$ & $121(61-277)$ \\
\hline Abatacept & 2710 & $28,158(18,677-37,653)$ & $99(63-162)$ \\
\hline Tocilizumab & 2182 & $28,920(15,782-42,081)$ & $79(34-187)$ \\
\hline Natalizumab & 1864 & $47,775(22,463-73,110)$ & $41(23-89)$ \\
\hline Cetuximab & 1451 & $80,792(15,351-146,256)$ & $19(9-103)$ \\
\hline Certolizumab pegol & 1446 & $34,460(11,632-50,322)$ & $45(17-163)$ \\
\hline Palivizumab & 1381 & $22,914(9139-36,697)$ & $63(34-167)$ \\
\hline Iplimumab & 1330 & $123,321(114,373-132,282)$ & $11(8-14)$ \\
\hline
\end{tabular}

$m A b$ monoclonal antibody, US\$ United States dollars, GBP Great British pound, max maximum, min minimum

${ }^{a} 2013-2018$ global sales were used to calculate the estimated monoclonal antibody yearly users. 2018 data are shown here

${ }^{\mathrm{b}}$ Maximum value is based on electronic supplementary Table S2, while minimum value is based on electronic supplementary Table S3, with 1 GBP converted to 1.524 US\$ 
Fig. 3 Ratio of total cases (monoclonal antibody-induced SCLE) against the annual estimated monoclonal antibody users as a percentage. Therapies utilising anti-tumour necrosis factor-a mechanisms are highlighted in yellow and anti-PD-1 drugs are shown in orange. SCLE subacute cutaneous lupus erythematosus, $P D-1$ programmed cell death-1

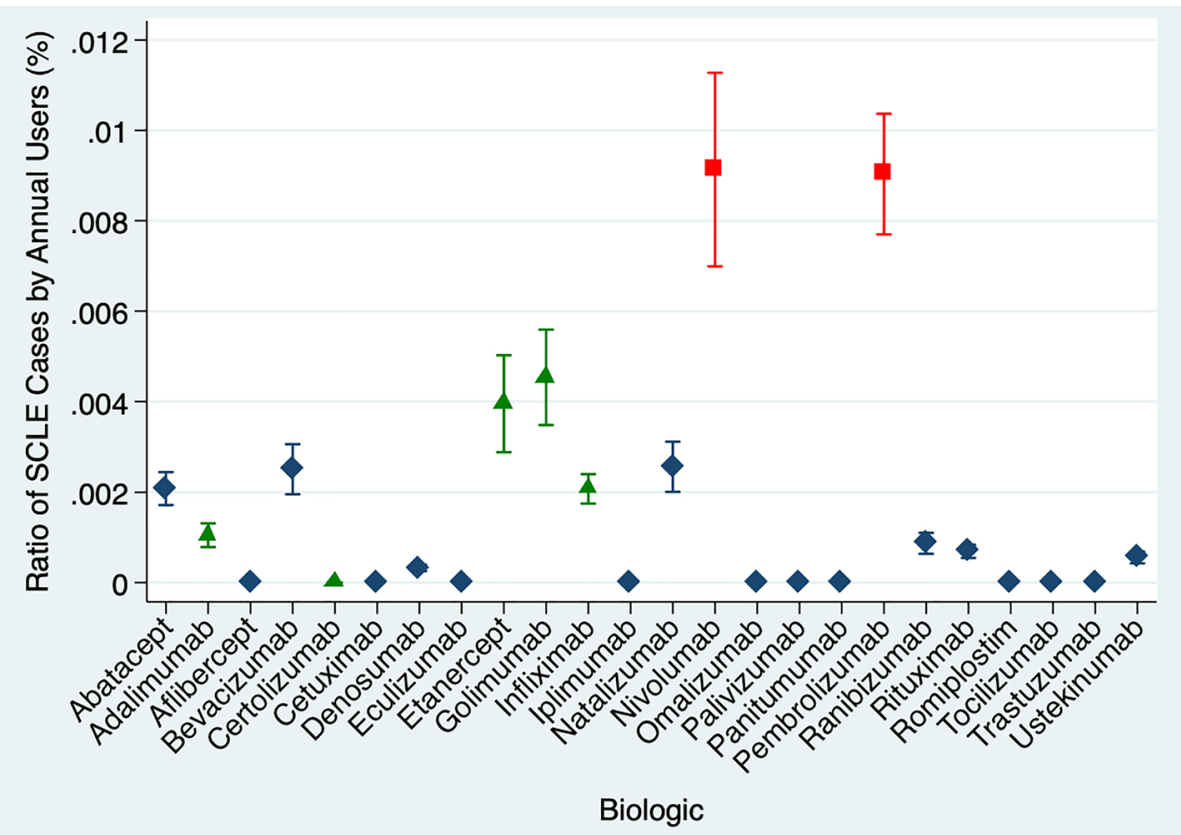

In isolation, the specificity of many criteria is limited [12, 37, 38]. Features delineating subacute and chronic forms of CLE include the presence of scarring/induration [37], duration of the rash, and the extent of histology findings. However, such features lack discriminatory boundaries for the spectrum of presentations $[36,37]$ and scarring does not present in the initial phases of the disease. Unlike typical adverse drug reactions, drug-induced cases of SCLE may present after an extended lag phase of drug exposure [14] and resolution of the condition may not necessarily follow drug cessation [39]. Some lesion types have been rarely considered as forms of SCLE, for example with gyrate, bullous or nodular appearances [40, 41]. In keeping with the EUSCLE categories [18], these lesion types were considered to be non-specific LE forms and were not included in our morphological criteria of SCLE. We opted for a specific set of phenotypes to promote clear and comparable use of diagnostic terms, aiming to assist in research and clinical management.

\subsection{Clinical Associations}

Previous reviews on the patient demographics of DI-SCLE and non-DI-SCLE cases found comparable characteristics as those identified in this review [42-45]. Similar to other patients with DI-SCLE, mAb-induced SCLE most commonly occurred in White female patients with a mean age of diagnosis between 40 and 65 years [14, 42], suggesting common susceptibility factors or simply reflecting the majority demographic of patients being administered these medications. The time course of mAb-induced SCLE was similar to a series of DI-SCLE where a median of 3-8 weeks to onset and 4-9 weeks to resolution was reported [43, 45].

For the variables considered, we did not find compelling evidence to suggest a distinct difference between the mAbinduced SCLE phenotype and DI-SCLE more generally. Compared with recent DI-SCLE studies [14, 43, 45, 46], this systematic review found a similar proportion of patients with detectable anti-Ro antibodies (62\% in this review vs. 72.1-100\%), ANA (85\% in this review vs. 64-100\%) and anti-dsDNA antibodies (27\% vs. 21\%) [45]. Anti-Ro antibodies are associated with drug-induced SLE in a minority of patients $(<5 \%$ [47]), however, consistent with the literature, we found a much higher proportion of seropositive patients in SCLE. A previous literature review reported a higher prevalence of anti-dsDNA antibodies in mAb-induced SCLE compared with DI-SCLE-91\% vs. 1\% [48], a finding not supported by our review. Compared with the series of 88 patients with DI-SCLE by Laurinaviciene et al. [45], the difference in anti-Ro and anti-dsDNA antibody status was not significant within the sample size $(p=0.2678$ and $p=0.5988$, respectively).

Patients with systemic features in association with mAbinduced SCLE exhibited a similarly mild course to that reported in DI-SCLE [19], with no cases of lupus nephritis or CNS involvement [42]. Lesions over the legs have been depicted as being more typical of DI-SCLE than idiopathic SCLE [43], which was supported by our finding of $30 \%$ of patients with leg involvement. The distribution alone suggests that photosensitivity may play less of a role in the pathophysiology of mAb-SCLE compared with classical SCLE. No cases with an explicit clinical SCLE diagnosis 
described lesions over the feet or the hands alone. In contrast, two patient cases that were excluded for non-specific lesion morphology showed lesions on the fingers and toes, with a dose onset that diverged from the IQR described [49, 50]. This suggests that lesions on the fingers or toes, in isolation, should prompt reconsideration of the diagnosis.

\subsection{Treatment}

Treatment modalities for CLE lack expert consensus or licencing [51], with extreme variation seen in surveys of dermatologists from Japan, Europe and the US [11]. Our analysis of the 49 patient cases with documented treatment confirmed this lack of standardised approach with a wide variety of treatment strategies used. There is a paucity of high-quality evidence for SCLE treatment [51]. At the time of writing, there were no active trials registered exclusively for SCLE on ClinicalTrials.gov [52] (last searched 6 April 2020). However, phase II trials are underway for janus kinase 1 (JAK1) inhibitors in CLE, and registries are being established to advance understanding.

Topical corticosteroids are considered to be the mainstay for limited cutaneous disease in CLE [11, 20, 51]. Topical calcineurin inhibitors have been suggested for corticosteroid-refractory SCLE [20]; however, a previous systematic review found such therapy to be less efficacious in SCLE compared with other CLE subtypes [53]. In terms of systemic options, DMARDs, specifically antimalarials, have long been used for their immunomodulating effects and avoidance of corticosteroid adverse effects [11]. The evidence base for this therapy in SCLE derives largely from one randomised blinded study of 12 patients in 1992 [51, 54], as well as clinical experience. Among the 19 patients who recovered fastest in our review (within 3 weeks), topical corticosteroids were most frequently used $(n=9)$; however, this rapid resolution likely reflects a milder disease presentation influencing clinicians in their management approach, as many other patients receiving topical corticosteroids and systemic agents showed a more prolonged course. Oral corticosteroids were widely used; however, recommendations suggest their use should be limited to severe disease [51] because of the frequency of adverse effects.

\section{4 mAb Use}

Given the rarity of SCLE, if there is a specific genetic, epigenetic, or pharmacokinetic context that predisposes a patient to mAb-induced SCLE, then greater use of a drug will heighten the likelihood of a predisposed patient receiving the triggering drug. However, we wanted to determine if the difference in case numbers reported for different mAbs simply reflected the differing number of yearly users.
Following modelling of yearly users, we identified that antiTNFa agents and checkpoint inhibitors were associated with a propensity for $\mathrm{mAb}$-SCLE relative to their use. Given the recent licencing of nivolumab and pembrolizumab, their high relative rates of SCLE are particularly stark.

Immune checkpoint inhibitors blocking PD-1 or CTLA-4 pathways are utilised to reduce immune tolerance of malignancy and T-cell anergy [55]; however, a loss of the inhibitory signals transmitted by such pathways may tip the balance towards T-cell-mediated inflammation and destruction of self-tissues [55]. Such an association between checkpoint inhibitors, CLE and other autoimmune dermatoses is increasingly being recognised. Further support for an association is illustrated by the lupus-like graft-versus-host syndrome in 2C-TCR-transgenic PD-1-deficient $\mathrm{H}-2^{\mathrm{b} / \mathrm{d}}$ mice, where skin lesions with dense inflammatory cell infiltrate in the dermis and liquefaction degeneration were observed [56]. The difference in SCLE cases associated with mAbs acting on PD-1 compared with CTLA-4 may be related to their primary site of action, with CTLA-4 limiting early T-cell responses, particularly in lymphoid tissues, and PD-1 limiting T-cell responses later in peripheral tissues [57]. While the propensity to immune-mediated adverse effects in patients taking checkpoint inhibitors is intuitive, further work is needed to understand why SCLE is induced in only a tiny proportion of patients receiving these therapies.

We considered whether the relatively high rate of SCLE associated with anti-TNFa agents could reflect a publication bias, with rheumatologists more likely to diagnose and publish cases attributable to drugs they frequently use. However, we found more cases were reported by dermatologists than rheumatologists, and such a trend was not consistent across other rheumatological therapies of rituximab, ustekinumab or tocilizumab (Fig. 3). Multiple previous studies have reported an association between anti-TNFa agents and the induction of CLE [47, 58]. TNFa is a cytokine involved in myriad signalling pathways of apoptosis, inflammation and immune regulation depending on the cellular context [59-61]. This wide array of cellular effects in different cell types may account for the conflicting findings of both the beneficial and antagonistic effects of TNFa on lupus in animal models [62, 63] and a small number of case reports [42, 64]. The significance of specific TNF pathways, and even anti-TNF mechanisms, are not well understood in CLE [65]. mAbs targeting TNFa do not only bind and sequester soluble cytokine but may also induce apoptosis [66-68], inhibit reverse signals from transmembrane TNFa [69] or enhance regulatory T-cell interactions [70]. Nonetheless in largerscale studies, the $-308 \mathrm{~A}$ TNFa promoter polymorphism has been associated with an increased risk of SCLE [71], and cutaneous TNF genes are significantly differentially expressed in CLE compared with controls [20]. 


\subsection{Limitations}

A multispecialty panel devised our inclusion criteria for the purpose of this review, therefore external validation is required. As most papers we included reported retrospective case series, this review was limited by incomplete data from reported cases. Some tests were not performed, however this is reflective of routine clinical care. Eight of the included sources came from conference abstracts, suggesting that grey literature was incorporated in the review; however, further hand searching would reduce publication bias further. The methodology has a language bias, with search strategies performed in the English language; we were unable to include non-European language papers in the screening process.

Annual mAb use was intended to provide a relative estimate of global use, however due to limited available prescription data of this kind, it is difficult to validate against existing data. The approximate annual costs utilised averages across two countries and cannot fully reflect the broad array of regimens used by physicians. Some mAb therapies have been licenced for many more years than others; for example, rituximab was licenced in 1997 . Therefore, using estimated patient users across 1 year underestimates exposure of patients to the older drugs, as it does not incorporate the decades of use. Given this, the relationship between checkpoint inhibitors and $\mathrm{mAb}$-induced SCLE is more striking as they have been licenced in the last decade.

\section{Conclusion}

Within the disease subtype of mAb-induced SCLE, there is great variability in serological findings, lesion distribution, treatment approach, and even the range of synonyms used for diagnostic terms. As the sheer complexity of the mechanisms governing tolerance and autoimmunity are further uncovered, clear phenotypic distinction and clarity of terms are vital to furthering understanding. To this end, we present the first systematic review of $\mathrm{mAb}$-induced SCLE.

Acknowledgements The authors are grateful for the support of Katharina Nucken in providing German translation assistance of the article. JS is supported by the National Institute for Health Research Biomedical Research Centre at the University of Oxford (NIHR-BRC-1215-20008).

Author Contributions $\mathrm{CB}$ is the overall guarantor for this work. EH and $\mathrm{CB}$ developed the search strategy. $\mathrm{CB}$, YC, JS and IS contributed to the study design. SH, BM, CB, SE and AC contributed to the development of the selection criteria. YC, IS, CB and RH performed abstract screening, with $\mathrm{CB}$ adjudicating any disparities. $\mathrm{CB}, \mathrm{RH}$ and $\mathrm{HW}$ performed the critical appraisal. $\mathrm{CB}, \mathrm{RH}, \mathrm{YC}$ and IS undertook data extraction. $\mathrm{CB}$, JS and YC drafted the manuscript. JS and CB calculated the annual estimated $\mathrm{mAb}$ use. All authors read and provided feedback on the final manuscript.

\section{Declarations}

Funding None.

Conflict of interest Christine Bolton, Yifan Chen, Ianthe R.M Schepel, Elinor Harriss, Silke C. Hofmann, Spencer Ellis, Alexander Clarke, Rachel Hawthorne, Helena Wace, Blanca Martin, Joel Smith declare there are no conflicts of interest.

Ethics approval None required.

Consent to participate Not applicable.

Consent for publication Not applicable.

Availability of data and material Provided in the electronic supplementary material and also from the corresponding author upon request.

Code availability Not applicable.

Open Access This article is licensed under a Creative Commons Attribution-NonCommercial 4.0 International License, which permits any non-commercial use, sharing, adaptation, distribution and reproduction in any medium or format, as long as you give appropriate credit to the original author(s) and the source, provide a link to the Creative Commons licence, and indicate if changes were made. The images or other third party material in this article are included in the article's Creative Commons licence, unless indicated otherwise in a credit line to the material. If material is not included in the article's Creative Commons licence and your intended use is not permitted by statutory regulation or exceeds the permitted use, you will need to obtain permission directly from the copyright holder. To view a copy of this licence, visit http://creativecommons.org/licenses/by-nc/4.0/.

\section{References}

1. Giovanni MA, Murray MF. Genome-first findings require precision phenotyping. Genet Med. 2018;20:1510-1.

2. Loscalzo J, Kohane I, Barabasi A-L. Human disease classification in the postgenomic era: a complex systems approach to human pathobiology. Mol Syst Biol. 2007;3:124.

3. Kohane IS. Deeper, longer phenotyping to accelerate the discovery of the genetic architectures of diseases. Genome Biol. 2014; $15: 115$.

4. Robinson PN, Mungall CJ, Haendel M. Capturing phenotypes for precision medicine. Cold Spring Harb Mol Case Stud. 2015;1:a000372.

5. Robinson PN, Köhler S, Bauer S, et al. The human phenotype ontology: a tool for annotating and analyzing human hereditary disease. Am J Hum Genet. 2008;83:610-5.

6. Ribero S, Sciascia S, Borradori L, et al. The cutaneous spectrum of lupus erythematosus. Clin Rev Allergy Immunol. 2017;53:291-305.

7. Jarukitsopa S, Hoganson DD, Crowson CS, et al. Epidemiology of systemic lupus erythematosus and cutaneous lupus erythematosus in a predominantly white population in the United States. Arthritis Care Res (Hoboken). 2015;67:817-28.

8. Patel P, Werth V. Cutaneous lupus erythematosus: a review. Dermatol Clin. 2002;20:373-85.

9. Watanabe T, Tsuchida T. Classification of lupus erythematosus based upon cutaneous manifestations. Dermatology. 1995;190:277-83. 
10. Sontheimer RD, Thomas JR, Gilliam JN. Subacute cutaneous lupus erythematosus: a cutaneous marker for a distinct lupus erythematosus subset. Arch Dermatol. 1979;115:1409-15.

11. Schultz HY, Dutz JP, Furukawa F, et al. From pathogenesis, epidemiology, and genetics to definitions, diagnosis, and treatments of cutaneous lupus erythematosus and dermatomyositis: a report from the 3rd international conference on cutaneous lupus erythematosus (ICCLE) 2013. J Investig Dermatol. 2015;135:7-12.

12. Srivastava M, Rencic A, Diglio G, et al. Drug-induced, Ro/ SSA-positive cutaneous lupus erythematosus. Arch Dermatol. 2003;139:45-9.

13. Sontheimer RD, Henderson CL, Grau RH, et al. Drug-induced subacute cutaneous lupus erythematosus: a paradigm for bedsideto-bench patient-oriented translational clinical investigation. Arch Dermatol Res. 2009;301:65-70.

14. Lowe G, Henderson CL, Grau RH, et al. A systematic review of drug-induced subacute cutaneous lupus erythematosus. Br J Dermatol. 2011;164:465-72.

15. Michaelis TC, Sontheimer RD, Lowe GC. An update in druginduced subacute cutaneous lupus erythematosus. Dermatol Online J. 2017;23:13030/qt55x42822.

16. Reed BR, Huff JC, Jones SK, et al. Subacute cutaneous lupus erythematosus associated with hydrochlorothiazide therapy. Ann Intern Med. 1985;103:49-51.

17. Bolton C, Mouyis K, Bhamra K, et al. The first case of natalizumab-induced subacute cutaenous lupus erythematosus. Rheumatology. 2020;59(6):1446-7.

18. Kuhn A, Kuehn E, Meuth AM, et al. Development of a Core Set Questionnaire by the European Society of Cutaneous Lupus Erythematosus (EUSCLE). Autoimmun Rev. 2009;8:702-12.

19. Sontheimer RD. Subacute cutaneous lupus erythematosus: 25 -year evolution of a prototypic subset (subphenotype) of lupus erythematosus defined by characteristic cutaneous, pathological, immunological, and genetic findings. Autoimmun Rev. 2005;4:253-63.

20. Sinha AA, Dey-Rao R. Pathogenesis of cutaneous lupus. In: Wallace DJ, Hahn BH, Crow MK, et al., editors. Dubois' lupus erythematosus and related syndromes. Philadelphia: Elsevier; 2018. p. 305-316.

21. Lee S, Harvey CP. Drug-induced systemic lupus erythematosus: a critical review. Semin Arthritis Rheum. 1975;5:83-103.

22. European Workshop on Skin Immune Mediated Inflammatory Diseases (SIMID). Conference abstracts-European workshop on SIMID. Verona, 24-26 April 2014, p 1-82.

23. Ecker DM, Jones SD, Levine HL. The therapeutic monoclonal antibody market. MAbs. 2015;7:9-14.

24. Philippidis A. Top 15 best-selling drugs of 2018. Genetic engineering and biotechnology news. 2019. https://www.genengnews .com/a-lists/top-15-best-selling-drugs-of-2018/. Accessed 29 July 2019.

25. Urquhart L. Top drugs and companies by sales in 2017. Nat Rev Drug Discov. 2018;17:232-232.

26. Office of the Assistant Secretary for Planning and Evaluation. Comparison of US and international prices for top medicare part b drugs by total expenditures. 2018. https://aspe.hhs.gov/system/ files/pdf/259996/ComparisonUSInternationalPricesTopSpendi ngPartBDrugs.pdf. Accessed 30 July 2019.

27. Hernandez I, Bott SW, Patel AS, et al. Pricing of monoclonal antibody therapies: higher if used for cancer? Am J Manag Care. 2018;24:109-12.

28. America's Health Insurance Plans. High-price drugs based upon estimated annual per-patient expenditure high-priced drugs: estimates of annual per-patient expenditures for 150 specialty medications. 2016. https://www.ahip.org/wp-content/uploads/2016/04/ HighPriceDrugsReport.pdf. Accessed 27 May 2020.
29. National Institute for Health and Clinical Excellence. Etanercept, infliximab and adalimumab for the treatment of psoriatic arthritis. NICE Guidance 2010. Technology appraisal guidance [TA199].

30. Bentley DD, Graves JE, Smith DI, et al. Efalizumab-induced subacute cutaneous lupus erythematosus. J Am Acad Dermatol. 2006;54:S242-S243243.

31. Vihinen P, Paija O, Kivisaari A, et al. Cutaneous lupus erythematosus after treatment with paclitaxel and bevacizumab for metastatic breast cancer: a case report. J Med Case Rep. 2011;5:243.

32. Brunasso A, Aberer W, Massone C, et al. Subacute lupus erythematosus during treatment with golimumab for seronegative rheumatoid arthritis. Lupus. 2014;23:201-3.

33. Marano AL, Clarke JM, Morse MA, et al. Subacute cutaneous lupus erythematosus and dermatomyositis associated with antiprogrammed cell death 1 therapy. Br J Dermatol. 2019;181:580-3.

34. Cura M. TNFalpha inhibitor induced lupus-like syndrome. Int $\mathbf{J}$ Dermatol. 2017;56:1308-9.

35. Andric M, Dixit S, Robaei D, et al. A case of subacute cutaneous lupus erythematosus as a result of ranibizumab (Lucentis) treatment. Indian J Ophthalmol. 2013;61:752.

36. Kuhn A, Landmann A. The classification and diagnosis of cutaneous lupus erythematosus. J Autoimmun. 2014;48-49:14-9.

37. David-Bajar KM, Bennion SD, DeSpain JD, et al. Clinical, histologic, and immunofluorescent distinctions between subacute cutaneous lupus erythematosus and discoid lupus erythematosus. J Investig Dermatol. 1992;99:251-7.

38. Sontheimer RD. Lichenoid tissue reaction/interface dermatitis: clinical and histological perspectives. J Investig Dermatol. 2009; 129:1088-99.

39. Vabre-Latre C-M, Bayle P, Marguery M-C, et al. Worsening of subacute lupus erythematosus induced by infliximab [in French]. Ann Dermatol Venereol. 2005;132:349-53.

40. Callen JP, Hughes AP, Kulp-Shorten C. Subacute cutaneous lupus erythematosus induced or exacerbated by terbinafine. Arch Dermatol. 2001;137:1196-8.

41. Perera GK, Black MM, McGibbon DH. Bullous subacute cutaneous lupus erythematosus. Clin Exp Dermatol. 2004;29:265-7.

42. Alniemi DT, Gutierrez A, Drage LA, et al. Subacute cutaneous lupus erythematosus. Mayo Clin Proc. 2017;92:406-14.

43. Marzano AV, Lazzari R, Polloni I, et al. Drug-induced subacute cutaneous lupus erythematosus: evidence for differences from its idiopathic counterpart. Br J Dermatol. 2011;165:335-41.

44. Grönhagen CM, Fored CM, Linder M, et al. Subacute cutaneous lupus erythematosus and its association with drugs: a populationbased matched case-control study of 234 patients in Sweden. $\mathrm{Br}$ J Dermatol. 2012;167:296-305.

45. Laurinaviciene R, Sandholdt LH, Bygum A. Drug-induced cutaneous lupus erythematosus: 88 new cases. Eur J Dermatol. 2017;27:28-33.

46. Biazar C, Sigges J, Patsinakidis N, et al. Cutaneous lupus erythematosus: First multicenter database analysis of 1002 patients from the European Society of Cutaneous Lupus Erythematosus (EUSCLE). Autoimmun Rev. 2013;12:444-54.

47. Dalle Vedove C, Simon JC, Girolomoni G. Drug-induced lupus erythematosus with emphasis on skin manifestations and the role of anti-TNF $\alpha$ agents. J der Dtsch Dermatologischen Gesellschaft. 2012;10:889-97.

48. Costa MF, Said NR, Zimmermann B. Drug-induced lupus due to anti-tumor necrosis factor $\alpha$ agents. Semin Arthritis Rheum. 2008;37:381-7.

49. Amarante CF, Acedo LMS, de Rabay FMO, et al. Drug-induced lupus with leukocytoclastic vasculitis: a rare expression associated with adalimumab. An Bras Dermatol. 2015;90:121-4.

50. Jee H, Kim S, Kim D, et al. Atypical cutaneous manifestation of lupus-like syndrome after etanercept treatment in seropositive rheumatoid arthritis: P17-42. J Dermatol. 2012;39:53-280. 
51. Kuhn A, Ruland V, Bonsmann G, et al. Cutaneous lupus erythematosus: update of therapeutic options: part II. J Am Acad Dermatol. 2011;65:e195-e213.

52. U.S National Library of Medicine. Search of: subacute cutaneous lupus-List Results-ClinicalTrials.gov. 2019. https:// clinicaltrials.gov/ct2/results?cond=subacute+cutaneous+lupus $\&$ term $=\&$ cntry $=\&$ state $=\&$ city $=\&$ dist $=\&$ Search $=$ Search. Accessed 23 July 2019.

53. Tzellos TG, Kouvelas D. Topical tacrolimus and pimecrolimus in the treatment of cutaneous lupus erythematosus: an evidencebased evaluation. Eur J Clin Pharmacol. 2008;64:337-41.

54. Ruzicka T, Sommerburg C, Goerz G, et al. Treatment of cutaneous lupus erythematosus with acitretin and hydroxychloroquine. Br J Dermatol. 1992;127:513-8.

55. Pardoll DM. The blockade of immune checkpoints in cancer immunotherapy. Nat Rev Cancer. 2012;12:252-64.

56. Nishimura H, Nose M, Hiai H, et al. Development of lupuslike autoimmune diseases by disruption of the PD-1 gene encoding an ITIM motif-carrying immunoreceptor. Immunity. 1999;11:141-51.

57. Buchbinder EI, Desai A. CTLA-4 and PD-1 pathways. Am J Clin Oncol. 2016;39:98-106.

58. Williams VL, Cohen PR. TNF alpha antagonist-induced lupus-like syndrome: report and review of the literature with implications for treatment with alternative TNF alpha antagonists. Int J Dermatol. 2011;50:619-25.

59. Sedger LM, McDermott MF. TNF and TNF-receptors: from mediators of cell death and inflammation to therapeutic giants - past, present and future. Cytokine Growth Factor Rev. 2014;25:453-72.

60. Qin J-Z, Bacon P, Panella J, et al. Low-dose UV-radiation sensitizes keratinocytes to TRAIL-induced apoptosis. J Cell Physiol. 2004; 200:155-166

61. Tartaglia LA, Rothe M, Hu Y-F, et al. Tumor necrosis factor's cytotoxic activity is signaled by the p55 TNF receptor. Cell. 1993;73:213-6.
62. Segal R, Dayan M, Zinger H, et al. Suppression of experimental systemic lupus erythematosus (SLE) in mice via TNF inhibition by an anti-TNF $\alpha$ monoclonal antibody and by pentoxiphylline. Lupus. 2001;10:23-31.

63. Brennan DC, Yui MA, Wuthrich RP, et al. Tumor necrosis factor and IL-1 in New Zealand Black/White mice. Enhanced gene expression and acceleration of renal injury. J Immunol. 1989;143:3470-5.

64. Norman R, Greenberg RG, Jackson JM. Case reports of etanercept in inflammatory dermatoses. J Am Acad Dermatol. 2006;54:S139-S142142.

65. Levine D, Switlyk SA, Gottlieb A. Cutaneous lupus erythematosus and anti-TNF-alpha therapy: a case report with review of the literature. J Drugs Dermatol. 2010;9:1283-7.

66. Eder P, Lykowska-Szuber L, Krela-Kazmierczak I, et al. The influence of infliximab and adalimumab on the expression of apoptosis-related proteins in lamina propria mononuclear cells and enterocytes in Crohn's disease-an immunohistochemical study. J Crohn's Colitis. 2013;7:706-16.

67. Shen C, Van Assche G, Rutgeerts P, et al. Caspase activation and apoptosis induction by adalimumab: demonstration in vitro and in vivo in a chimeric mouse model. Inflamm Bowel Dis. 2006;12:22-8.

68. Malaviya R, Sun Y, Tan JK, et al. Etanercept induces apoptosis of dermal dendritic cells in psoriatic plaques of responding patients. J Am Acad Dermatol. 2006;55:590-7.

69. Al Izzi M, Reda A. Cutaneous lupus erythematosus induced by golimumab therapy. Int J Rheum Dis. 2017;20:2159-61.

70. Nguyen DX, Ehrenstein MR. Anti-TNF drives regulatory T cell expansion by paradoxically promoting membrane TNF-TNF-RII binding in rheumatoid arthritis. J Exp Med. 2016;213:1241-53.

71. Werth VP, Zhang W, Dortzbach K, et al. Association of a promoter polymorphism of tumor necrosis factor- $\alpha$ with subacute cutaneous lupus erythematosus and distinct photoregulation of transcription1. J Investig Dermatol. 2000;115:726-30.

\section{Affiliations}

\section{Chrissy Bolton 1,2,11 $\cdot$ Yifan Chen ${ }^{2} \cdot$ Rachel Hawthorne $^{3}$ - lanthe R. M. Schepel ${ }^{2}$. Elinor Harriss ${ }^{4} \cdot$ Silke C. Hofmann $^{5}$. Spencer Ellis ${ }^{6} \cdot$ Alexander Clarke $^{7} \cdot$ Helena Wace $^{8} \cdot$ Blanca Martin $^{9} \cdot$ Joel Smith $^{10}$}

1 University College London, University College London Hospitals NHS Foundation Trust, London, UK

2 Medical Sciences Division, University of Oxford, Oxford, UK

3 John Radcliffe Hospital, Oxford University Hospitals NHS Trust, Oxford, UK

4 Bodleian Health Care Libraries, The Knowledge Centre, Oxford University Old Road Campus Research Building, Oxford, UK

5 Department of Dermatology, Allergology and Dermatosurgery, HELIOS University Hospital Wuppertal, University of Witten/Herdecke, Wuppertal, Germany

6 Lister Hospital, East and North Herts NHS Trust, Stevenage, UK
7 The Kennedy Institute of Rheumatology, University of Oxford, Oxford, UK

8 Addenbrookes Hospital, Cambridge University Hospitals NHS Foundation Trust, Cambridge, UK

9 Department of Dermatopathology, St John's Institute of Dermatology, St Thomas' Hospital, London, UK

10 Nuffield Department of Population Health, University of Oxford, Oxford, UK

11 Translational Gastroenterology Unit, Experimental Medicine, University of Oxford, John Radcliffe Hospital, Oxford OX3 9DU, UK 